\title{
Hallucinogen Dependence, Episodic Use
}

National Cancer Institute

\section{Source}

National Cancer Institute. Hallucinogen Dependence, Episodic Use. NCI Thesaurus. Code C35385.

Physical and psychological dependence on hallucinogenics, which is associated with a pattern of episodic use. 